\title{
Managing Aspect Orderings to Support Multiple Quality Concerns
}

\author{
Bedir Tekinerdoğan \\ Bilkent University \\ Dept. of Computer Engineering \\ 06800 Bilkent, Ankara, Turkey
}

bedir@cs.bilkent.edu.tr

\author{
Ersin Er \\ Hacettepe University \\ Dept. of Computer Engineering \\ 06800 Beytepe, Ankara, Turkey
}

ersin.er@cs.hacettepe.edu.tr

\begin{abstract}
When multiple aspects are composed undesired behavior may emerge due to the interference of aspects. Different interference management approaches have been proposed including detection and resolution of the conflicting aspects. It appears that the majority of the existing approaches have basically focused on functional correctness, whereby orderings of aspects are evaluated with respect to assumed contractual specification. Although functional correctness is an important quality concern also other quality concerns such as evolvability, reuse and reliability can demand a specific ordering. As such, the resulting possible set of orderings might need to be further reduced. In this paper we discuss the impact of other quality concerns than functional correctness, on the required orderings of aspects. Based on a domain analysis of existing approaches we provide a feature model and complementary to this a metamodel for defining aspect interference management approaches for multiple quality concerns.
\end{abstract}

\section{Categories and Subject Descriptors}

D.2.2 [Software Engineering]: Design Tools and Techniques.

\section{General Terms}

Design, Documentation, Performance, Verification

\section{Keywords}

Aspect interference, metamodeling, aspect ordering, quality concerns

\section{INTRODUCTION}

Aspect-Oriented Software Development (AOSD) provides abstractions to separate and modularize crosscutting concerns into aspects and compose these later in the base code [1]. If multiple aspects are composed, aspects can interact in the base code. This does not pose a problem if the aspects are orthogonal to each other, that is, if their order of processing does not impact the behavior of the interacting aspects. It has been shown, though, that the interaction of aspects can lead to undesired behavior due

Permission to make digital or hard copies of all or part of this work for personal or classroom use is granted without fee provided that copies are not made or distributed for profit or commercial advantage and that copies bear this notice and the full citation on the first page. To copy otherwise, or republish, to post on servers or to redistribute to lists, requires prior specific permission and/or a fee.

AOM'09, March 2, 2009, Charlottesville, VA, USA.

Copyright 2009 ACM 978-1-60558-451-5/09/03...\$5.00. to the so-called interference of aspects [9][12]. Aspects interfere with each other if the order of processing the aspects is semantically relevant for the final result [5].

In recent years several studies have been carried out to address the aspect interference problem and a relatively broad insight has been gained on this topic. To cope with this problem several aspect interference detection and resolution approaches have been proposed. The proposed techniques for solving the aspect interference problem are usually dependent on the type of interference [12]. For example, static analysis techniques have been proposed to identify potential shared join-points of the aspects. To detect indirect interaction of aspects through data variables, dataflow analysis and tracing techniques can be applied. To detect interference based on semantic properties contractual specifications of aspects together with model checking techniques can be applied [12].

Despite the different interpretations of interference and the different techniques that are proposed, we can observe that all these approaches aim to order the composition of aspects explicitly to prevent undesired interference. A further analysis of the literature shows that the majority of the existing aspect interference approaches have mainly focused on functional correctness as a key motivation for aspect ordering. This means that either implicitly or explicitly it is assumed that an aspect has to adhere to some contractual specifications that define the semantic properties of the aspect.

Yet, although functional correctness is an important concern, if not the most important one, it appears that the ordering of aspects might also be of importance for different quality concerns such as evolvability, reuse, availability and performance. This means that aspects might (also) need to be ordered or reordered for these quality concerns. The main theme of this paper, as such, is that for ordering aspects not only functional correctness but each relevant quality concern should be explicitly and separately addressed. We think that this observation can further support the research on aspect composition, aspect interference and aspect interference detection and resolution problems.

Based on the existing research on aspect interference problem we first provide a domain model that defines the space of the aspect interference problem and the proposed techniques. The domain model will be presented as a feature model and does not only summarize existing work but may also help to detect new problems and aspect interference management approaches. Further, using a case study and a set of example scenarios we will define the motivation for ordering aspects for multiple quality 
concerns. Finally, we provide a metamodel and a generic process for defining aspect interference management approaches for multiple quality concerns.

The outline of the paper is structured as follows: In section 2 we provide the feature model for aspect interference problem and the related techniques. In section 3 we show the impact of aspect ordering on concerns other than functional correctness. In section 4 we define the metamodel for ordering aspects for multiple quality concerns, and define the process for applying the metamodel. Section 5 will provide the related work. Finally, section 6 provides the conclusions.

\section{ASPECT INTERACTION}

In recent years, several researchers have focused on the aspect interference problem, and this has resulted in several approaches and herewith a better understanding of the problem. To depict the space of the problem we will define feature diagrams for Aspect Composition, Relation among Aspects, Aspect Interference and Inference Detection. A feature diagram is a tree in which the root represents the domain concept being described and the remaining nodes denote features. Features of a concept can be mandatory, alternative, or optional [2]. The feature diagrams that we present in the following are the result of a thorough domain analysis to the existing aspect interference management approaches.

The first and top level feature diagram is the one for Aspect Composition, which is depicted in Figure 1. Aspect Composition concept can be characterized using the features Composition Time, Composition Scheme, and Composition Result. Composition Time refers to the time of weaving the aspect, which can be basically at compile time, load time and run-time. Composition Scheme refers to whether the aspects are composed sequentially or concurrently [4]. Composition Result defines whether the aspects interact or not. The interaction might be direct on shared joinpoints or indirect through data members. Further, the interaction might lead to interference.

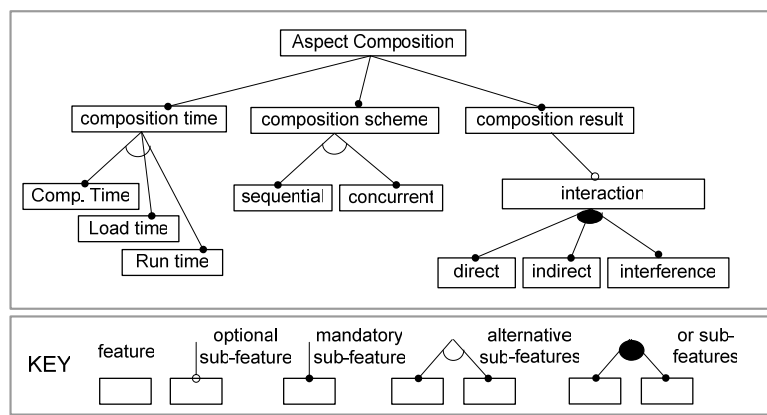

Figure 1. Top-level feature diagram of aspect composition

When aspects interact with each other it is important to know the possible relations among these aspects. Figure 2 defines the feature diagram for Aspectual Element Relations. Aspectual Element is either an aspect or advice. This is to denote that several interference management approaches consider composition of aspectual elements either at the granularity level of aspects and/or at the granularity level of advices. Aspectual elements have a relation with the base code (application), which can be spectative, regulative or invasive [8]. Aspects are spectative if they only query the state of the base system but do not change it. Aspects are regulative if they can alter the control flow of the base system. Finally, invasive aspects can alter both the control flow and the state of the base system. Invasive aspects can be further characterized by augmentation, narrowing, or replacement [11]. This means that aspectual elements can either augment the base code or narrow its functionality or replace it all together.

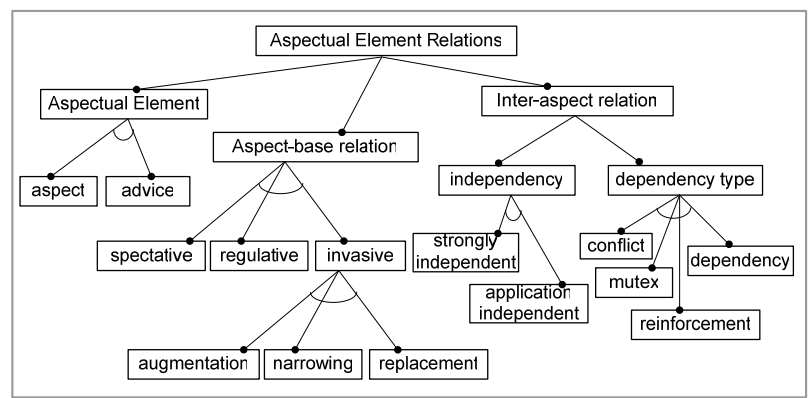

Figure 2. Feature diagram for aspect relations

The feature Inter-Aspect Relation defines the relation among aspects in an interaction. Aspectual elements can be dependent or independent from each other. In case of independency we can distinguish between strong independency and application independency [3]. In case of strong independency the aspects are independent for all programs. Application independence relates to independence of aspects for a given particular program. This implies that the same set of aspects could be dependent for a different program.

The feature Dependency Type defines the way aspects depend on each other. In principle four different dependency types can be distinguished, conflict, mutex, reinforcement, and dependency [12]. Aspects conflict with each other if they negatively affect each other's behavior. Mutex implies that the aspects cannot be composed together. Reinforcement occurs if an aspect positively influences the functionality of another aspect. Dependency implies that an aspect requires being composed together with another aspect.

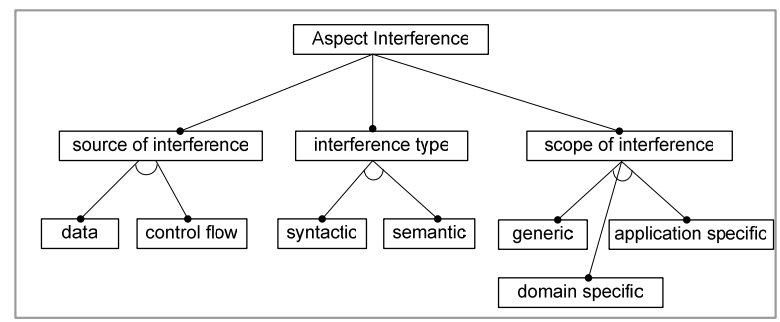

Figure 3. Feature diagram of aspect interference

Figure 3 represents the feature diagram for Aspect Interference which can be characterized using three sub-features source of interference type, interference type, and scope of interference. Source of interference can be either data or control flow related [7][9]. Interference type is either syntactic or semantic. Scope of interference can be generic, domain specific or application specific [5].

Figure 4 represents the feature diagram for Inference Detection which is characterized by three features time of detection, technique and aspect interference. The latter one is reused from the feature diagram as presented in Figure 3. The feature time of detection defines the time when the interference is detected. Basically, we can distinguish among pre-weaving, weaving time and post-weaving time. The feature approach defines the applied 
approaches for detecting interferences. It includes three subfeatures analysis scheme, target of analysis and technique. The analysis scheme can be done using static analysis [8] without executing the program. However, some interference problems cannot be detected statically and for these dynamic analysis can be applied. Hereby the interference is detected while executing the program or part of the program. The target of analysis could be either the interference related to data or control flow, corresponding to dataflow analysis and control flow analysis respectively. The feature method defines particular approaches to detect interference problems. These methods range from theorem proving to resource modeling approaches.

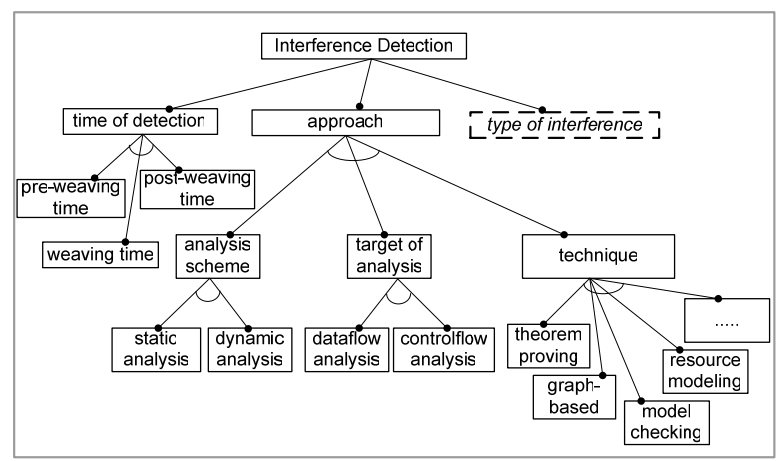

Figure 4. Feature diagram for conflict detection

\section{ASPECT ORDERING FOR OTHER QUALITY CONCERNS}

\subsection{Quality Concerns}

In the following we will show that also other quality concerns than functional correctness might demand a specific ordering. For this, let us first consider the feature diagram in Figure 5 for ordering aspectual elements for quality concerns.

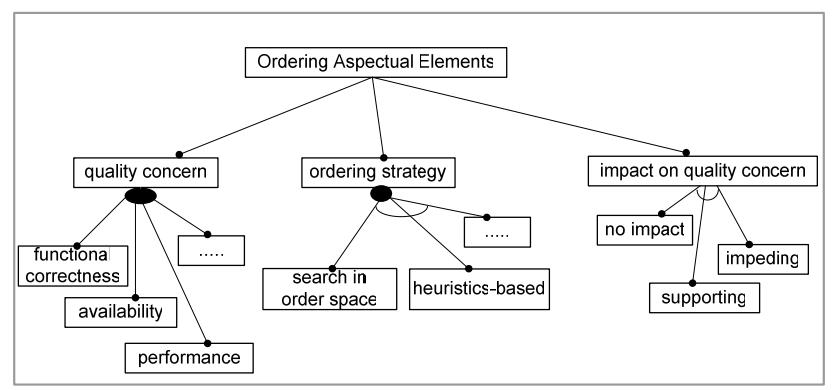

Figure 5. Feature diagram for ordering aspectual elements for quality concerns

Ordering Aspectual Elements can be done for various quality concerns such as functional correctness, availability and performance. We will discuss these in the following subsection. The aspects might need to be ordered according to some strategy. This could be through search in a order space, using heuristicsbased strategy or any other strategy. In fact, every ordering of aspects can have an impact on the considered quality concerns. We distinguish the following three impacts of ordering aspectual elements on a quality concern:

- $\quad$ no impact, the ordering does not matter.
- impeding, the specific ordering has a negative impact on the quality concern. For example, it might result in incorrect situation (correctness), lower performance or decrease availability.

- $\quad$ supporting, the specific ordering has a positive impact on the quality concern. For example, it might result in better performance, better availability or performance.

\subsection{Example Scenarios}

In the following subsections we will provide examples of quality concerns that require a specific ordering of aspects.

\subsubsection{Example: Ordering for Availability}

In general availability indicates the degree to which a system is available. When composing aspects, the order of the included aspects should not unnecessarily reduce the availability of the system. Let us discuss how the order can impede the availability. In general availability might be impeded due to failures in the system. The formula for availability is given as follows:

$$
\text { Availability }=\text { MTTF } /(M T T F+M T T R)
$$

MTTF and MTTR stand for the mean time to failure and the mean time to recover, respectively. To maximize the availability of the overall system, MTTF of separate modules must be kept high and MTTR must be kept low. An important heuristic might be that for having a low MTTR it is necessary to perform the recovery actions as soon as the failure is detected, and do not execute additional tasks that will be undone by recovery actions anyhow. This might imply that aspect Recovery needs to be invoked earlier than other aspects in order not to waste time for imposing other aspects that will be undone by the recovery aspect later on. As such, considering the orderings of aspects from availability perspective it makes sense to put the recovery aspect as early as possible. Note that other orderings where Recovery aspect is not put early on, might still be valid from the functional correctness concern perspective. The reasoning about Availability will further restrict the orderings. This is not only of importance to increase availability but has also a practical benefit in dramatically reducing the number of feasible ordering alternatives.

\subsubsection{Example: Ordering for Resource Usage Optimization}

Very often resource usage plays an important role in programs, and effort is spent to optimize the programs to that the resources are used efficiently. In this context, the ordering of aspects might have a direct impact on the resource usage. For this example let us consider the two aspects, Transaction Management and Authorization. Transaction Management ensures consistency of a system before and after the execution of certain operations which are considered as atomic units of work. Authorization is a process that establishes whether an authenticated user has sufficient permissions to access certain resources. Both Transaction Management and Authorization aspects can be considered as spectative aspects as defined in Figure 2, and as such do not change state variables in the system. Moreover, unless it is explicitly stated for a system, they do not interfere in any way from a functional correctness perspective. So the order of execution of Transaction Management and Authorization do not lead to a conflicting situation with respect to correctness criteria. However, when we consider Resource Usage Optimization as a 
quality concern, then it may be more appropriate to execute Authorization aspect first. The reason for this is that Authorization aspect can conditionally interrupt the execution of the aspect (ordering) chain in case of unauthorized access. For such a case, if Authorization is executed after Transaction Management, the system resources will be wasted by initiating an unnecessary transaction.

\subsubsection{Example: Ordering for Robustness and Evolvability}

In general every system has to cope with evolutionary requirements. One of the key concerns in such situations is to anticipate on the changing requirements and define a robust system. In aspect-oriented systems we can encounter both the evolution of base code and the evolution of aspects. If we are dealing with a single aspect, then the need for evolution of base code, might require the change of the pointcuts of the corresponding aspect. This is usually referred to as the fragile pointcut problem. Several techniques have been proposed to support the robustness of the pointcuts and to avoid the fragility of aspects. However, if we are dealing with a composition of aspects then this problem might have a larger impact. In case one or more aspects in the aspect ordering evolves, then this might have a further impact on the robustness of pointcuts of other aspects in the chain. In this perspective, we can term the problem as fragile ordering of aspects, which can be defined as the situation in which the aspect ordering needs to be broken due to evolution of either the base code or the aspect code. This is not an imaginary problem, because as we have seen before, aspects might have a dependency relation with each other. To release or relieve this problem, aspects might need to be reordered.

\section{METAMODEL FOR ORDERING ASPECTS}

In the previous sections we have provided a domain model for aspect compositions and the interference problems and approaches. In addition we have indicated the need for considering multiple quality concerns. In this section we provide a metamodel that can be used to define aspect interference management approaches and the generic process for applying it.

\subsection{Metamodel}

The metamodel is depicted in Figure 6. Hereby, AspectualElement is an implementation of crosscutting unit, which may be of different granularity such as an aspect or an advice. AspectualElements are managed by AspectualElementManager which adds, removes or updates aspectual elements to the AspectualElementList. The AspectualElementList is ordered by the OrderManager to manage the interference of aspectual elements. OrderManager considers one or more QualityConcerns, which is modeled by QualityModel. OrderManager uses InterferenceModel and AspectualModel to order AspectualElements. AspectualModel represents the abstraction of AspectualElement with respect to QualityModel. While QualityModel represents a general abstraction of QualityConcern, AspectualModel represents a specific abstraction of AspectualElement with respect to the general QualityModel. InterferenceModel uses QualityModel and AspectualModel to define either the feasible or the conflicting orderings of AspectualElements with respect to the considered QualityConcerns. For this InterferenceModel can use different techniques such as rules, predicates, regular expressions, automata, or temporal logic [5].

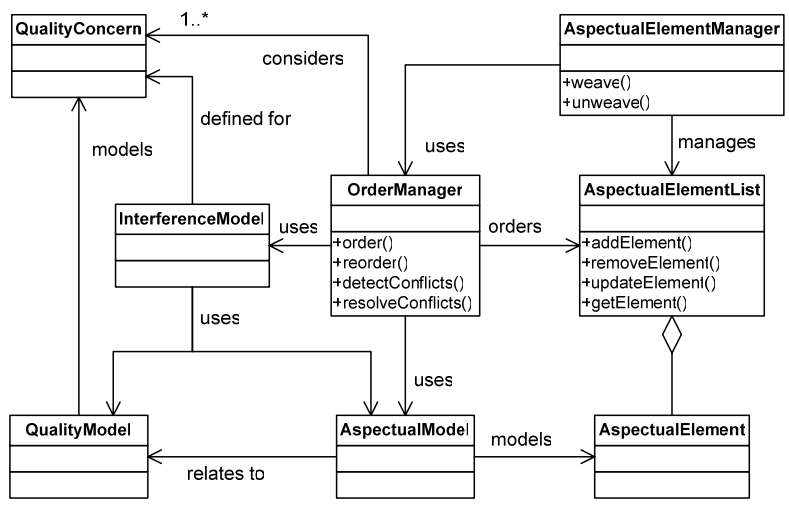

Figure 6. Metamodel for Ordering Aspects based on multiple quality concerns

\subsection{Process for applying metamodel}

The metamodel of Figure 6 defines the general concepts that can be used to define multiple aspect interference approaches. Obviously, to define a particular approach each concept should be specified concretely. In this paper we do not elaborate on defining a specific approach. Instead, we will define a generic process that can be instantiated to define a concrete aspect interference approach. The process is depicted in Figure 7.

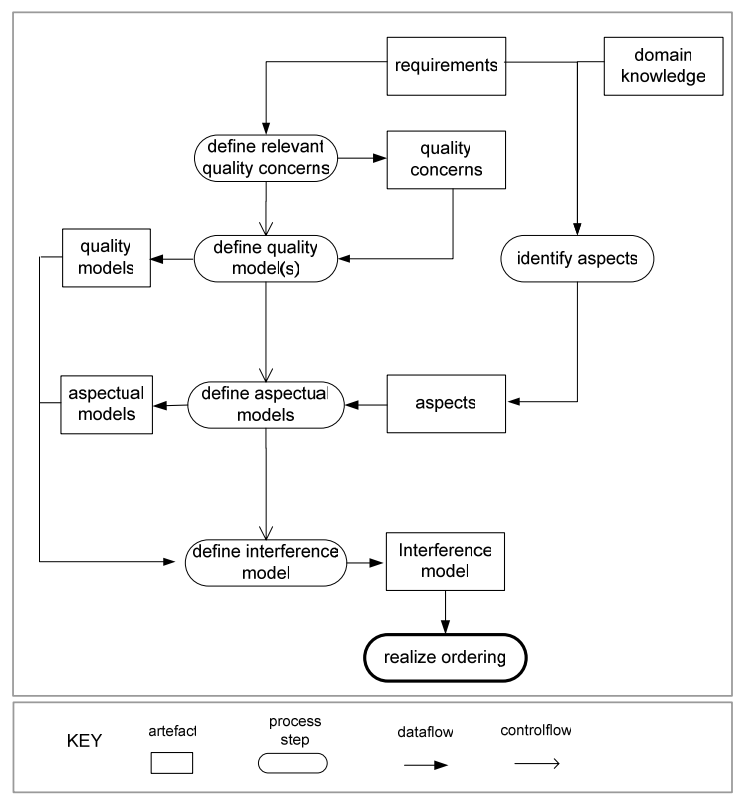

Figure 7. Generic process for aspect interference management for multiple quality concerns

The first step in the process is the identification of quality concerns which are derived from the requirements. Based on the identified quality concerns, quality models are defined. The step define aspectual models takes as input the quality models and the aspects and as such result in aspectual models. Aspects are derived from the step identify aspects which uses requirements and domain knowledge. The step define interference model takes as input the quality models and the aspectual models and provides 
an interference model. The interference model can be used for realizing the ordering of aspects in the application.

Note that this generic process aims also to cover the existing aspect interference approaches in the literature. In addition we can define a specific process based on this generic process. For this we need to select the specific features from the feature diagram in section 2. For example we could define an interference management system for the quality concerns correctness and availability. We could consider different kind of aspects, adopt specific interference modeling techniques, use different ordering strategies etc.

\section{RELATED WORK}

As a result of a domain analysis process in this paper we have provided feature models that represent the key features of aspect interference management approaches. To the best of our knowledge this has not been defined before. In the literature we could identify several surveys on aspect interference management approaches such as defined in [7]. We have analyzed these and other publications on specific approaches to define the domain model.

To define the aspect orderings it is very important to know the properties of aspects. In this way the analysis on the interaction of aspects will be supported. For analyzing the aspects the Network of Excellence on AOSD deliverable "A domain analysis of key concerns - known and new candidates" [10] provides an invaluable resource of key aspects. The deliverable includes both traditionally known crosscutting concerns (persistence, security, context awareness and mobility) and more recent candidate crosscutting concerns (agent technology and coordination).

Obviously one of the related key domains that are relevant for the approach that we presented in this paper is the domain of quality attributes. A lot has been published about different quality concerns and its measurement. In this context, ISO 9126 is an international standard for the evaluation of software quality [6]. The standard is divided into four parts: quality model, external metrics, internal metrics and quality in use metrics. The quality model established in the first part of the standard, classifies software quality in a structured set of characteristics and subcharacteristics.

\section{CONCLUSIONS}

If aspects are composed together in a system, they may interact. If aspects interact, they may semantically interfere. Interference of aspects is bad because they can violate functional correctness. Despite most of the aspect interference management approaches have indeed focused on functional correctness, it appears that aspect interactions are also important for other quality concerns such as availability, performance and evolvability. Since most quality concerns are non-functional and cannot be easily expressed as functional requirements, they need to be addressed explicitly to define feasible aspect orderings. This was the main theme of this paper. When considering aspect orderings we have to take multiple quality concerns into account. To support our statement we have first provided the domain model for aspect interaction and aspect interference and showed that despite the broad knowledge on aspect interference management, the current trend is basically on functional correctness. We have used a number of example scenarios to show the requirements of different quality concerns on aspect orderings. We have given the examples for correctness, availability, resource usage and evolvability. Obviously other quality concerns could be provided here. For this a more focused analysis to each quality concern is required. We have defined a metamodel that aims to reflect the existing approaches for managing aspect interactions and which can be used to define new aspect interference management approaches. To support the definition of specific aspect interference management approaches we have defined a generic process for realizing the metamodel.

We hope that our study paves the way for a further study towards the impact of orderings of aspect to other quality concerns than just functional correctness. In particular, the requirements of different quality concerns on aspect ordering, needs further investigation. When we are dealing with multiple quality concerns, sooner or later we have also to consider the trade-off among these quality concerns. We have not elaborated on this trade-off analysis of quality concerns yet, but we consider this as our future work. Finally, since aspect interference management is hard to define manually, we will focus on tool development based on the metamodel.

\section{REFERENCES}

[1] AOSD-Europe. European Network of Excellence on AspectOriented Software Development. European Commission grant IST-2-004349.

[2] Czarnecki, K. and Eisenecker. U. Generative Programming: Methods, Tools, and Applications, Addison Wesley, 2000.

[3] Douence, R. and Fradet, P. A framework for the detection and resolution of aspect interactions. In GPCE: ACM SIGPLAN/ SIGSOFT Conference, GPCE 2002, Lecture Notes in Computer Science, Pittsburgh, US, October,6 2002.

[4] Douence, R., Le Botlan, D., Noyé,J., and Südholt, M. Concurrent aspects. In Proc.of the 5th international Conference on Generative Programming and Component Engineering, Portland, USA, October 22 - 26, 2006).

[5] Dürr, P., Staijen,T., Bergmans, L. and Aksit, M. Reasoning about semantic conflicts between aspects. In EIWAS '05: The 2nd European Interactive Workshop on Aspects in Software, Brussel, Belgium, September, 1-2, 2005.

[6] International Organization for Standardization. Software Engineering - Product Quality - Part 1: Quality Model. ISO/IEC 9126-1:2001(E), Geneva, Switzerland, 2001.

[7] S. Katz et. al. Detecting Interference among Aspects, NoE AOSD Deliverable, D116, 2007.

[8] Katz, S. A Survey of Verification and Static Analysis for Aspects, AOSD Europe Milestone M8.1, July 2005.

[9] Leavens, G. T. and Clifton, C. Foundations of aspectoriented languages workshops. In Foundations of AspectOriented Languages Workshop, AOSD, 2003-2008.

[10] Loughran, N., et al. A domain analysis of key concerns known and new candidates, NoE AOSD Deliverable D

[11] Rinard, M., Salcianu,A. and Bugrara, S. A classification system and analysis for interactions in aspect-oriented programs. In Foundations of Software Engineering (FOSE). ACM, Oct. 2004.

[12] Sanen, F., Truyen, E., Joosen, W., Loughran, N., Rashid, A. Jackson, A. Nedos, A. and Clarke, S. Study on interaction issues. AOSD-Europe Deliverable 44. March 2006. 